\title{
Safety investigation on rail and road vehicles exposed to cross-wind: wind tunnel tests and multi-body simulations
}

\author{
F. Cheli, R. Corradi, D. Rocchi, E. Sabbioni \& G. Tomasini \\ Mechanical Engineering Department, Politecnico di Milano, Italy
}

\begin{abstract}
A methodology aimed at evaluating the limit safety conditions of road and rail vehicles when exposed to cross wind is presented in this paper. The procedure integrates wind-tunnel experimental tests and numerical simulations with Multi-Body (MB) vehicle models. The main results of the proposed methodology are the vehicle Critical Wind Curves, representing the combination between wind and vehicle speed, corresponding to the overcoming of the vehicle safety limit.
\end{abstract}

Keywords: MB simulations, wind tunnel testing, safety, aerodynamic forces.

\section{Introduction}

Over the years the effects of strong winds on road and rail vehicles have become of increasing concern for transportation system operators. The main risk associated with cross wind is the vehicle rollover, which is particularly critical when the vehicle exits a tunnel or when the vehicle runs in a curve on exposed sites such as viaducts, embankments or long-span bridges. Traffic disruption, economic loss, injury, mortality and risks connected with the transported goods, such as hydrocarbons, toxic, flammable and explosive substances, etc. result as a consequence.

Aim of the work is to describe the methodology developed for assessing of the limit safety conditions of road and rail vehicles in terms of Critical Wind Curves (CWC) that represent the combination of vehicle speed and wind mean speed which leads to the overcoming of the safety limits. The methodology integrates wind-tunnel experimental tests and numerical simulations with MultiBody (MB) vehicle models. The experimental analysis (wind tunnel testing) 
allows one to evaluate both the static aerodynamic coefficients, from the measurement of the static component of the aerodynamic forces, and the aerodynamic admittance function. The vehicle transient dynamic response when exposed to cross-winds is then assessed through simulations with MB models of both rail and road vehicles. The unsteady aerodynamic loads due to the turbulent wind acting on the vehicle are calculated through an algorithm, based on both static aerodynamic coefficients and admittance function, measured during wind tunnel testing on reduced scale vehicle models.

\section{Numerical experimental methodology}

The proposed numerical-experimental methodology for evaluating the Critical Wind Curves of a road/rail vehicle can be divided into two phases:

- experimental measurements performed during wind tunnel tests on scale models;

- numerical analysis to evaluate the dynamic response of a vehicle exposed to turbulent cross wind.

Figure 1 shows the flow chart of the overall numerical-experimental procedure. The inputs to the numerical algorithm for the evaluation of the aerodynamic forces due to the cross wind action on the vehicle are:

1. static aerodynamic coefficients of the vehicle;

2. aerodynamic admittance function;

3. turbulent wind speed distribution as a function of time and space.

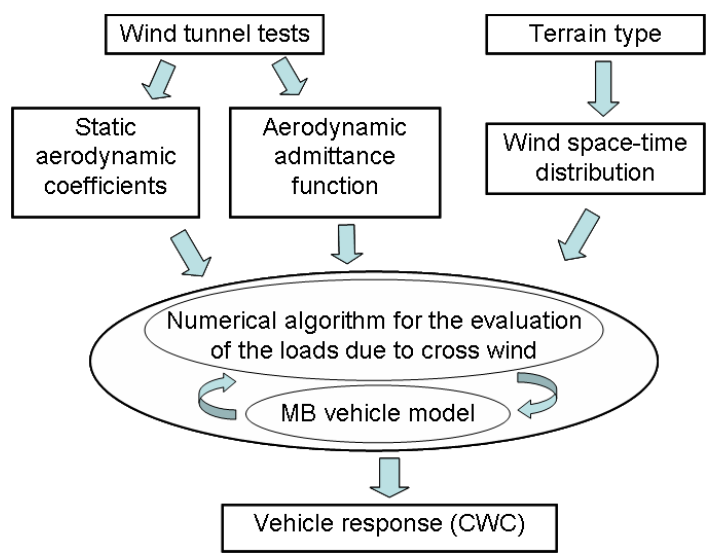

Figure 1: Experimental-numerical methodology flow-chart.

With reference to points 1 . and 2., the experimental tests allow to evaluate both the static aerodynamic coefficients, from the measurement of the static component of the aerodynamic forces, and the aerodynamic admittance function. This function accounts for the spatial correlation of pressures at any two points on the vehicle's surface and it represents a modifying adjustment of the ideal case of a vehicle enveloped by turbulent wind with full spatial correlation [1]. 
About the third point, since turbulent wind is a random process, a time-space distribution of the wind speed can be simulated for a given mean wind speed and terrain type, known the statistical properties of the atmospheric boundary layer: wind speed Power Spectral Density (PSD), turbulence intensity, integral length scales [2]. The numerical algorithm for the evaluation of the aerodynamic loads is directly interfaced with a MB model for the calculation of the vehicle dynamic behaviour (Figure 1). The limit safety conditions are then determined by evaluating the vehicle Critical Wind Curves (CWC).

\section{Wind tunnel tests}

The first part of the proposed methodology consists in performing wind tunnel tests on scale vehicle models in order to experimentally measure the vehicle mean aerodynamic coefficients and the vehicle aerodynamic admittance function (inputs of the numerical algorithm for the evaluation of the aerodynamic forces, Figure 1). Different kind of vehicles have been tested along the years: ETR500, ETR480, EMUV250 and IC train UIC-Z1 control trailer coach (Figures 7 and 8), for rail vehicles, and lorry, tank, articulated vehicle (Figure 5), for road heavy vehicles.

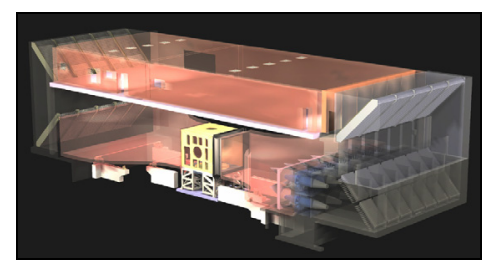

Figure 2: $\quad$ Politecnico di Milano wind tunnel.

Table 1: $\quad$ Main characteristics of the Politecnico di Milano wind tunnel.

\begin{tabular}{|l|l|l|l|}
\hline \multicolumn{2}{|l|}{ Max. installed power } & 1.5 & {$[\mathrm{MW}]$} \\
\hline Global dimensions & $50 \times 15 \times 15$ & {$[\mathrm{mxmxm}]$} \\
\hline Test section & Dimensions [m] & Max. vel. [m/s] & $\Delta \mathrm{u} / \mathrm{u}[\%]$ \\
\hline Civil & $14 \times 4$ & 18 & $< \pm 2$ \\
\hline Aeronautical & $4 \times 4$ & 60 & $< \pm 0.2$ \\
\hline
\end{tabular}

Tests are usually carried out in the civil section (see Table 1) of the Politecnico di Milano wind tunnel (see Figure 2), which allows testing large scale models, together with significant reduction of undesirable edge and blockage effects.

Different flow conditions can be reproduced during the tests in order to evaluate the effect of the wind characteristics on the aerodynamic coefficients. Low turbulence flow is obtained in the standard wind tunnel operating conditions. It is characterised by a uniform vertical profile of the mean wind velocity (see Figure 3). By properly adding roughness elements and spires before 
the test section (Figure 6), different atmospheric boundary layer can also be reproduced (turbulence intensity up to $30 \%$ ). As an example, Figure 4 shows the comparison between the wind speed vertical profile experimentally measured during full scale tests and the one reproduced in the wind tunnel within the WEATHER European project [13].

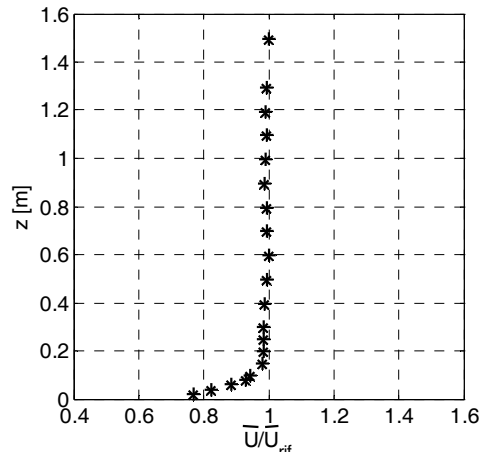

Figure 3: Low turbulence flow: normalized vertical profile of mean wind speed.

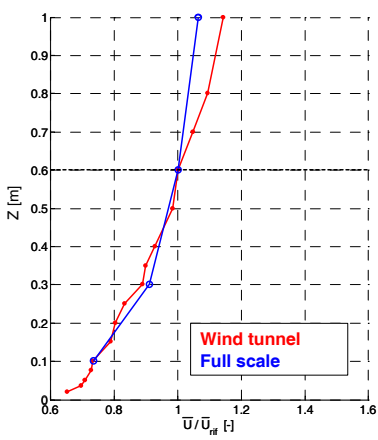

Figure 4: High turbulent flow: normalized vertical profile of the mean wind. Comparison with full scale test.

According to the TSI standard, the reference configuration for the static aerodynamic coefficients evaluation is the flat ground (Figure 6a and Figure 7). However, in order to take into account the vehicle real working conditions, several scenarios can be reproduced (viaduct, embankment, presence of solid porous fences, see Figures 5-7). Tests are carried out considering both upwind and downwind conditions.
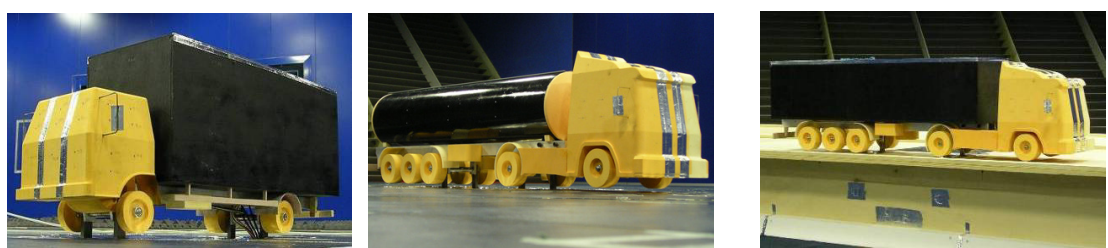

Figure 5: Tested road heavy vehicles (lorry, tank, articulated vehicle).

The aerodynamic forces and moments in the three directions are measured by means of a six-components dynamometric balance, set under the model and connected to the vehicle in correspondence of the wheels. The wind speed is measured by means of both Pitot tubes for the mean wind speed and a Cobra probe, for the measurement of the statistical characteristics of the turbulent wind (turbulence intensity, wind Power Spectral Density function, integral length 
scale, [1]). Some preliminary tests have also been carried out with a moving train (tunnel exit, Figure 8), in order to confirm that the aerodynamic coefficients measured in static and in dynamic conditions are the same for the same relative wind speed (hypothesis of the numerical algorithm for the aerodynamic loads evaluation). During these tests, a six components dynamometric balance has been placed between the boogies and the carbody in order to measure the aerodynamic forces and moments acting on the carbody itself.

Once the aerodynamic forces and moments $\left(F_{i}\right.$ and $\left.M_{i}\right)$ and the mean wind speed $\bar{U}$ have been measured, the corresponding aerodynamic coefficients can be evaluated from the quasi-steady theory $[1,4,5]$ :

$$
F_{i}=\frac{1}{2} \rho_{a} A C_{F i} \bar{U}^{2} ; \quad M_{i}=\frac{1}{2} \rho_{a} A h C_{M i} \bar{U}^{2} \quad(i=x, y, z)
$$

where $A$ stands for the lateral surface of the carbody, $h$ is a reference height and $\rho_{a}$ is the air density.

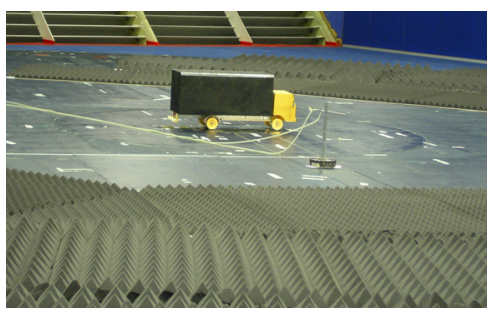

(a)

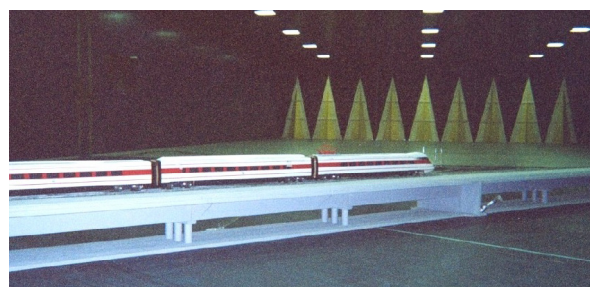

(b)

Figure 6: Boundary layer simulations: (a) Lorry model on flat ground. (b) Train model on viaduct.

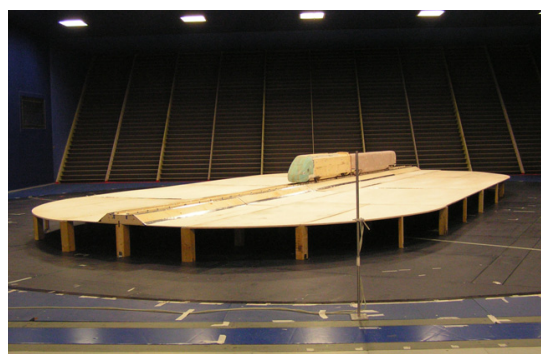

Figure 7: EMUV250 on flat ground.

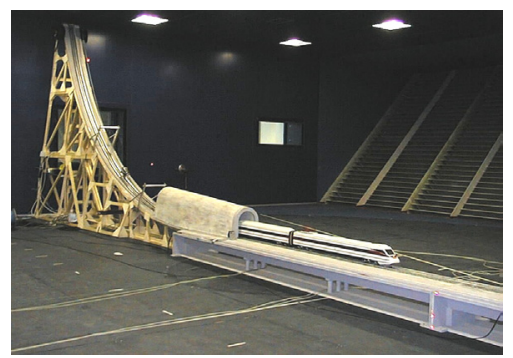

Figure 8: Dynamic test (ETR470): tunnel exit.

As an example, the influence of the scenario (flat ground and embankment) is analysed in Figure 9 for the ETR500 train. The overturning moment coefficient is shown as a function of the yaw angle $\alpha$.

The tests carried out in atmospheric boundary layer conditions also allow to identify the vehicle aerodynamic admittance function $\mathrm{H}$ [1]: the square of the aerodynamic admittance function is evaluated [4] as the non-dimensional ratio 
between the PSD of the measured aerodynamic force and the PSD of the aerodynamic force evaluated with the quasi-static theory, starting from the wind speed in the same experimental tests and the static aerodynamic coefficients (eq. (1)). As an example, the ETR500 aerodynamic admittance function is shown in Figure 10 as a function of the adimensional frequency $f^{x} L_{U} / \bar{U}$, where $f$ is the frequency and ${ }^{x} L_{U}$ is the longitudinal integral scale.

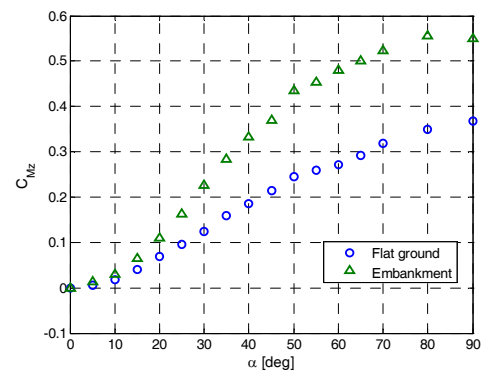

Figure 9: ETR500: overturning moment coefficient, influence of the scenario.

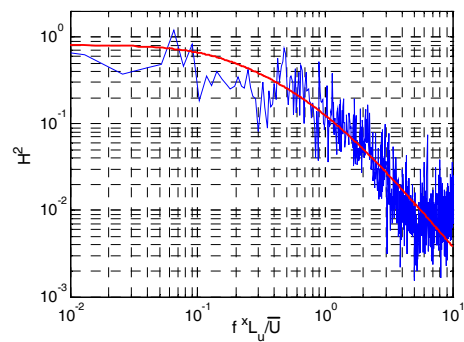

Figure 10: ETR 500: admittance function; experimental data and interpolating function.

\section{The numerical algorithm for wind loads evaluation}

A numerical algorithm has been set up for the aerodynamic loads evaluation. The adopted approach is based on the corrected quasi-steady theory, a classic approach for many applications [2]. This theory consists in applying the quasisteady theory (eq. (1)), rigorous for the calculation of the aerodynamic force mean value, and in correcting it, in the frequency domain, by the admittance function [4,5]: thus it represents a modifying adjustment of the ideal case of a vehicle enveloped by turbulent wind with full spatial correlation.

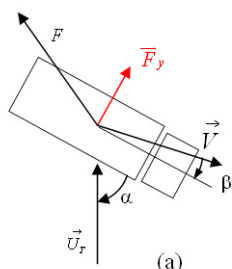

(a)

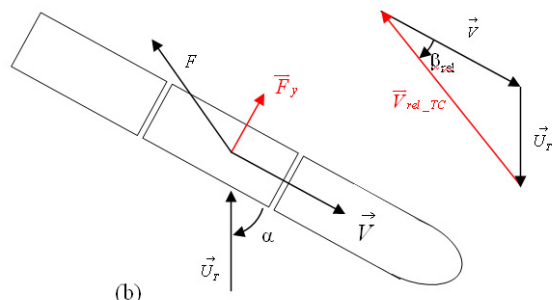

(b)

Figure 11: Corrected relative vehicle-wind velocity $V_{\text {rel_TC }}$ and related angle of attack $\beta_{\text {rel }}$ : (a) road vehicles, (b) rail vehicles. 
It is to point out that, for rail vehicles, being the trajectory imposed, the relative angle of attack ( $\beta_{\text {rel }}$, see Figure 11$)$ is determined once the wind direction (angle $\alpha$ ) and the track geometry are known. Thus the time history of the aerodynamic loads (input of the MB vehicle model) can be calculated in a pre-processing phase. Of course this is not possible for road vehicles, since the vehicle path (and consequently the relative angle of attack) depend on the external forces acting on it and on the driver response. In this case, the numerical algorithm for aerodynamic forces calculation is directly interfaced to the MB vehicle model (see Figure 1): during the integration process, at each time step, it gets as an input, from the dynamic model, the vehicle speed and the vehicle sideslip angle $\beta$ (that allows the evaluation of the vehicle trajectory, see Figure 11 ) and it gives as an output, to the vehicle model, the instantaneous value of the aerodynamic loads.

\section{Vehicle MB models}

The vehicle dynamic response to cross wind forces is evaluated through MB rail and road vehicle models developed by the Mechanical Engineering Department of Politecnico di Milano.

\subsection{Rail vehicle model}

In order to simulate the dynamic response of a rail vehicle subjected to cross wind, the algorithm for the aerodynamic loads calculation, has been implemented in A.D.Tre.S., a rail vehicle dynamic simulation program [3], developed by the Mechanical Engineering Department of Politecnico di Milano. A.D.Tre.S. is suitable for simulating the motion of a railway vehicle running on tangent track and curve, also taking into account the deformation of track and substructure and the rail and wheels surface corrugation. The vehicle model is composed of a car body, two bogies and four wheelsets, introduced as rigid or flexible bodies, by means of modal superposition approach [3]. Car body, bogies and wheelsets are linked one to each other by means of the elastic and damping elements reproducing the primary and secondary suspensions (see Figure 12). The simulation code accounts for the motion of a train-track system in both vertical and lateral direction, while the longitudinal motion of the vehicle is assumed to take place with constant velocity. The vehicle equations of motion are written with respect to a moving reference system, travelling with constant speed along the ideal track centreline.
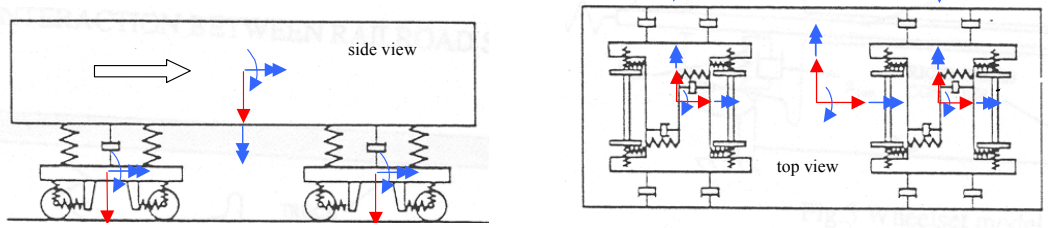

Figure 12: The rail vehicle model. 
The contact model is based on a preliminary geometrical analysis on the measured wheel and rail profiles. Contact parameters such as rolling radius and contact angle are reported in table form, as functions of wheel-rail lateral displacement. The geometrical analysis allows also to determine the number of the potential contact points for a given wheel-rail lateral displacement. Lateral and longitudinal creepages are computed and tangential and longitudinal forces at each active contact are obtained according to Shen, Hedrick and Elkins formulation [3].

The equations of motion are integrated in time domain, by means of a numerical step by step procedure [3]. In the present analysis, the simplifying assumptions of "infinitely rigid" track and vehicle rigid bodies were made.

\subsection{Road vehicle model}

In the case of road vehicle dynamic simulation, the algorithm for wind loads evaluation has been interfaced with a 14 d.o.f. vehicle model developed by the Mechanical Engineering Department of Politecnico di Milano [9,10]. The vehicle is considered as composed by five rigid bodies: the vehicle chassis (the sprung mass), having 6 d.o.f., and the four unsprung masses (the four wheels) each characterised by wheel rotation and a vertical movement. The vehicle chassis and the unsprung masses are connected through the suspension system, while the unsprung masses are linked to the ground by means of a spring-damper element, representing the tires radial stiffness and damping (see Figure 13). The suspensions have been introduced through multi-dimensional tables in order to take into account their elasto-kinematic behaviour. The engine is introduced through its characteristic curve, i.e. driving torque versus angular speed, while the deformability of the driveline is modelled through a first order system. The tires behaviour is described using MF-Tyre model version 2002 [11].

Steer angle, brake pressure, throttle valve position and gear are all regarded as imposed inputs characterising a particular manoeuvre and they can be either introduced by the user in pre-processing phase (open loop manoeuvres) or computed during the simulation by a driver model (closed loop manoeuvres).

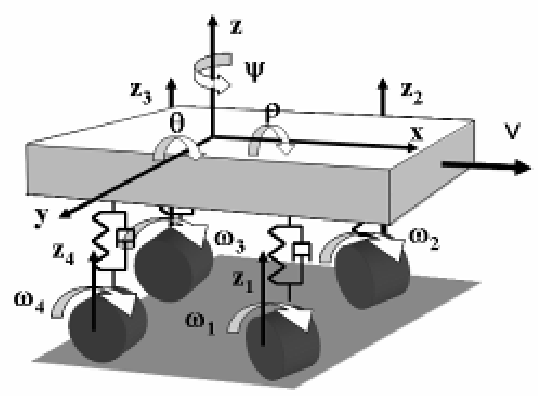

Figure 13: The 14 d.o.f. road vehicle model.

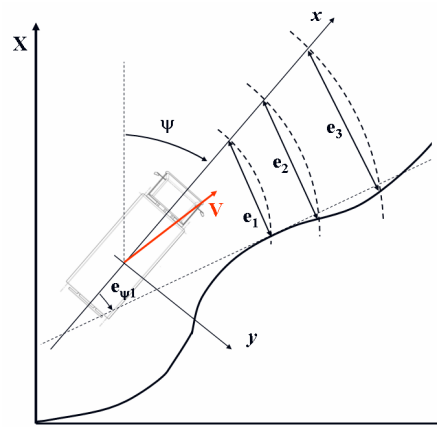

Figure 14: The road vehicle driver model. 
In order to obtain comparable results (wind forces acting on the vehicle depending both on the vehicle trajectory and on the wind speed time-space distribution, section 4), a driver model has been implemented to complete the simulation tool. The steering angle is determined through a PID (ProportionalIntegrative-Derivative) regulator acting on the error between the actual and the desired vehicle trajectory at three preview points $\left(\mathrm{e}_{1}, \mathrm{e}_{2}, \mathrm{e}_{3}\right.$ in Figure 14). In the steering angle calculation the nearest preview point is the most weighted.

\section{Simulation results}

The developed simulation tool consisting of the algorithm for the aerodynamic loads calculation and the MB vehicle model, can be used to evaluate the safety limit of the vehicle for each running condition. The index used to determine the Critical Wind Curves is the overturning coefficient (the Prudhomme criterion or the Nadal's coefficient can be considered as alternative indexes for defining the CWCs for rail vehicles [4]), defined as:

$$
\eta=\frac{\sum_{i}\left|Q_{\text {left }}-Q_{\text {right }}\right|}{\sum_{i}\left(Q_{\text {left }}+Q_{\text {right }}\right)}<\eta_{\text {lim }}=0.9 \quad(i=\text { bogie } \text { or axle })
$$

where $Q$ are the vertical loads acting on the wheels of each boogie/axle.

By comparing the time histories of the index with the corresponding limit value, it is possible to find whether the combination of vehicle speed $\mathrm{V}$ and gust wind speed $U_{\text {lim }}$ corresponds or not to the overcoming of the safety limit for the considered vehicle: the critical wind curves (CWC) represent the gust wind speed $U_{\text {lim }}$ that leads to the overcoming of the safety index as a function of the vehicle speed $V$.

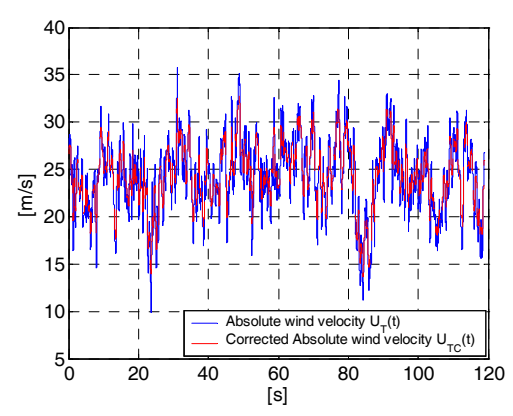

Figure 15: Tangent track simulation: time history of the wind speed, $\mathrm{I}_{U}=19 \%,{ }^{\mathrm{x}} \mathrm{L}_{\mathrm{U}}=90 \mathrm{~m}$ (input of the $\mathrm{MB}$ vehicle model).
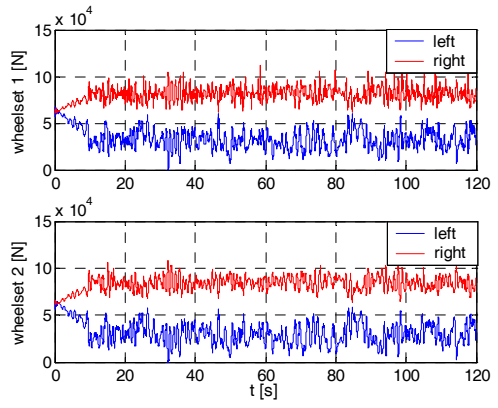

Figure 16: Tangent track simulation: time history of the vertical loads, $\mathrm{I}_{\mathrm{U}}=19 \%$, ${ }^{\mathrm{x}} \mathrm{L}_{\mathrm{U}}=90 \mathrm{~m}$ (output of the MB vehicle model). 
Figures 15 and 16 show an example of input and output time histories. A rail vehicle running in a tangent track and it is subjected to a turbulent wind having the following characteristics: $\bar{U}=25 \mathrm{~m} / \mathrm{s}$, turbulence intensity $\mathrm{I}_{\mathrm{u}}=19 \%$ and integral length scale ${ }^{x} L_{u}=90 m$. More in particular, Figure 15 shows the cross wind speed time history (input of the MB vehicle model), while in Figure 16 the time histories of the wheel vertical loads are reported (output of the MB model). Since the wind is coming from the left, a load transfer occurs from the left to the right wheels.

Starting from the simulation results, the safety index and the CWCs can thus be evaluated. Since turbulent wind is a random process, a statistical analysis can be performed: the resulting $\mathrm{CWC}$ can be evaluated as the mean value of several simulations carried out for different random generations of the turbulent wind speed given as an input to the $\mathrm{MB}$ vehicle model. As an example, Figure 17 shows the mean CWC and the relative dispersion band obtained for a train on flat track during a tangent track simulation (blue triangles). The mean CWC obtained through the proposed methodology (blue triangles) is compared with the one obtained using the TSI methodology (red crosses). As it can be seen, the TSI CWC is within the dispersion band.

The influence of several overall parameters can be taken into account and evaluated through the proposed methodology: the deformability of brides [12], the wind turbulence intensity, the considered scenario (flat ground, viaduct embankment, [4,5]), the track/road irregularity [4], etc. In Figure 18, the influence of the scenario during a tangent track simulation with a lorry is evaluated. As can be noticed, the flat ground is always the less critical situation. At low vehicle speed the embankment and the viaduct present the same trend, while at high vehicle speed the embankment seems to be the most critical condition. This is due to the fact that the wind flow is accelerated by the trapezoidal cross-section of the embankment.
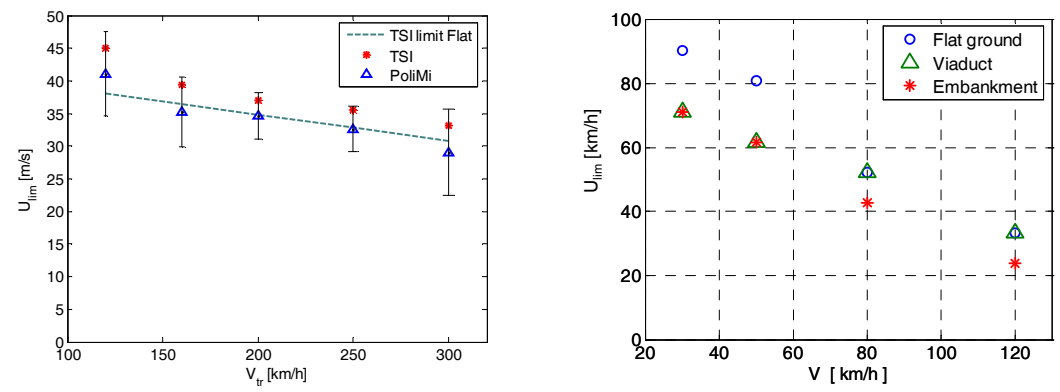

Figure 17: CWC: comparison between the PoliMi and Figure 18: CWC: influence of the TSI methodology, for a train, flat ground, tangent scenario. Lorry, tangent track. 


\section{Concluding remarks}

In the present paper a numerical-experimental methodology for the evaluation of vehicle limit safety conditions when exposed to cross wind has been presented, applicable to both rail and road vehicles. Wind tunnel experimental tests are needed in order to evaluate the vehicle aerodynamic coefficients in different operating conditions and to identify the vehicle admittance function. These are the inputs to the numerical algorithm for the turbulent wind loads evaluation (based on the corrected quasi-static theory), which is directly interfaced with MB $\mathrm{road} / \mathrm{rail}$ vehicle models. Through this simulation tool it is thus possible to assess the vehicle dynamics when exposed to cross wind and finally evaluate the safety limit conditions in terms of Critical Wind Curves. The proposed methodology has proved to be a useful tool for the design of on board vehicle controllers and infrastructure alert systems.

\section{References}

[1] Simiu, E., Scanlan, R.H., 1986, Wind effects on structures, New York, Wiley-Interscience.

[2] Cheli, F., Curami, A., Bocciolone, M., Zasso, A., 1991, Wind measurements on the Humber Bridge and numerical simulation, 8-th Int. Conference on Wind Engineering ICWE, London, Canada.

[3] Bruni, S., Collina, A., Diana, G. and Vanolo, P.: Lateral Dynamics Of A Railway Vehicle In Tangent Track And Curve: Tests And Simulations. Proceedings of 16-th IAVSD Symposium, Vol. 33 pp 464-477 (1999).

[4] Cheli F., Corradi R., Diana G., Tomasini G., A Numerical-Experimental Approach to Evaluate the Aerodynamic Effects on Rail Vehicle Dynamics, 18th IAVSD Symposium August 24 to 30, 2003.

[5] F. Cheli, P. Belforte, S. Melzi, E. Sabbioni, G. Tomasini: A numericalexperimental approach for evaluating cross wind aerodynamic effects on heavy vehicles, Proc. of XIX IAVSD Symposium, Milano, Italy, 29 August-2 September 2005.

[6] Bocciolone, M., Cheli, F., Corradi, R., Diana, G. and Tomasini, G.: Wind Tunnel Tests For The Identification Of The Aerodynamic Forces On Rail Vehicles. Proceedings of 11th ICWE - International Conference on Wind Engineering, June 2003.

[7] S. Bruni, F. Cheli, E. Sabbioni, S. Sciacca, G. Tomasini, Studio sperimentale delle caratteristiche aerodinamiche di veicoli stradali pesanti, IX Convegno IN-VENTO, Pescara, Italy, 19-22 June 2006.

[8] Cooper R. (1984), Atmospheric Turbulence with respect to moving ground vehicles, Journal of wind engineering and industrial aerodynamics, vol. 17, 215-238

[9] F. Cheli, E. Leo, S. Melzi, F. Mancosu, E. Giangiulio, D. Arosio, 2006, Implementation of a 14 d.o.f. model for the prediction of vehicle dynamics and is interaction with active control systems, Tire Technology Expo, Stutgart, Germany, 2006. 
[10] F. Cheli, E. Leo, S. Melzi, F. Mancosu, A 14 d.o.f. model for evaluation of vehicle's dynamics, International Journal of Mechanics and Control, Vol. 6, No. 2, pp. 19-30, 2005.

[11] H. B. Pacejka, Tyre and Vehicle Dynamics, Butterworth and Heinemann Editions, 2002.

[12] F. Cheli, A. Collina, E. Leo, F. Resta, G. Tomasini, Numericalexperimental methodology for runnability analysis and wind-bridgevehicle interaction study, III ECCM Conference, Lisbon, Portugal, June 2006.

[13] D. Delaunay, C. J. Baker, F. Cheli, H. Morvan et al., Development of wind alarm systems for road and rail vehicles: presentation of the WEATHER project, $13^{\text {th }}$ Int. Road Weather Conference, $25^{\text {th }}-27^{\text {th }}$ March 2006. 